05

\title{
Ферритовые пленки с повышенной стабильностью частоты ферромагнитного резонанса
}

\author{
(C) В.В. Шагаев, Тун Тун Лин
}

МГТУ им. Н.Э. Баумана, Калужский филииал, 248000 Калуга, Россия

e-mail: shagaev_vv@rambler.ru

(Поступило в Редакцию 8 июня 2016 г.)

Сформулирована задача, в которой требуется обеспечить устойчивость частоты магнитных колебаний ферритовой пленки при одновременном изменении значений двух магнитных параметров. Предложен метод стабилизации частоты однородного ферромагнитного резонанса при малых отклонениях эффективной намагниченности и поля кристаллографической магнитной анизотропии феррита от исходных значений. Выведено уравнение, которое в линейном приближении связывает вариацию частоты с вариациями магнитных параметров. Исследована возможность обращения в нуль коэффициентов при вариациях магнитных параметров. Показано, что такая возможность может быть реализована выбором кристаллографической ориентации пленки, напряженности и направления намагничивающего поля. В этом случае сдвиг частоты определятся квадратичной формой от вариаций магнитных параметров. Приведен пример расчета квадратичной формы. Проведено сравнение оптимизированной пленки с пленкой, у которой сдвиг частоты определяется линейной зависимостью от вариаций ее магнитных параметров.

DOI: 10.21883/JTF.2017.03.44242.1956

\section{Введение}

Спектр колебаний намагниченности монокристаллической ферритовой пленки зависит от значений магнитных параметров феррита, важнейшими из которых являются намагниченность насыщения $\left(4 \pi M_{0}\right)$, поле кристаллографической магнитной анизотропии $\left(H_{c}\right)$, и для пленок, выращенных на подложках, поле одноосной магнитной анизотропии $\left(H_{u}\right)[1]$. В ряде случаев вместо параметров $4 \pi M_{0}$ и $H_{u}$ можно использовать один комбинированный параметр - эффективную намагниченность $\left(4 \pi M_{\text {eff }}\right)$. Изменение параметров $4 \pi M_{\text {eff }}$ и $H_{c}$ приводит к смещению определяемых ими частот. Это смещение в линейном по изменениям $\delta\left(4 \pi M_{\text {eff }}\right)$ и $\delta H_{c}$ приближении можно рассчитать по уравнению

$$
\delta f=a \delta\left(4 \pi M_{\mathrm{eff}}\right)+b \delta H_{c},
$$

где $a=d f / d\left(4 \pi M_{\text {eff }}\right)$ и $b=d f / d H_{c}$. Условие стабильности частоты можно представить в виде $\delta f=0$. Есть два варианта выполнения условия. В первом - постоянство частоты достигается взаимной компенсацией слагаемых в правой части формулы (1). Во втором в нуль обращается каждое из слагаемых, и тогда $a=0$ и $b=0$. В этом случае в отличие от первого варианта условие $\delta f=0$ будет выполнено при любых значениях $\delta\left(4 \pi M_{\text {eff }}\right)$ и $\delta H_{c}$.

Первый вариант был подробно исследован в работах [2-4]. В этих работах рассчитаны температурные коэффициенты частот магнитостатических волн. В частности, исходя из условия термостабильности частот, была рассмотрена возможность взаимной компенсации температурных зависимостей намагниченности насыщения и поля анизотропии.
Второй вариант в научной литературе не описан. Именно ему посвящена настоящая работа. С практической точки зрения второй вариант интересен тем, что позволяет стабилизировать частоту независимо от природы изменения магнитных параметров феррита. Такие изменения могут быть связаны с температурой, с химическим составом, с упругими напряжениями.

\section{Разработка модели}

Предлагаемый способ был опробован в модели пленки кубического феррита при решении задачи по стабилизации частоты однородного ферромагнитного резонанса (ФМР) (рис. 1.)

Используемые обозначения: $\mathbf{M}_{0}$ - вектор намагниченности феррита, $\mathbf{H}_{e}$ - вектор напряженности внеш-

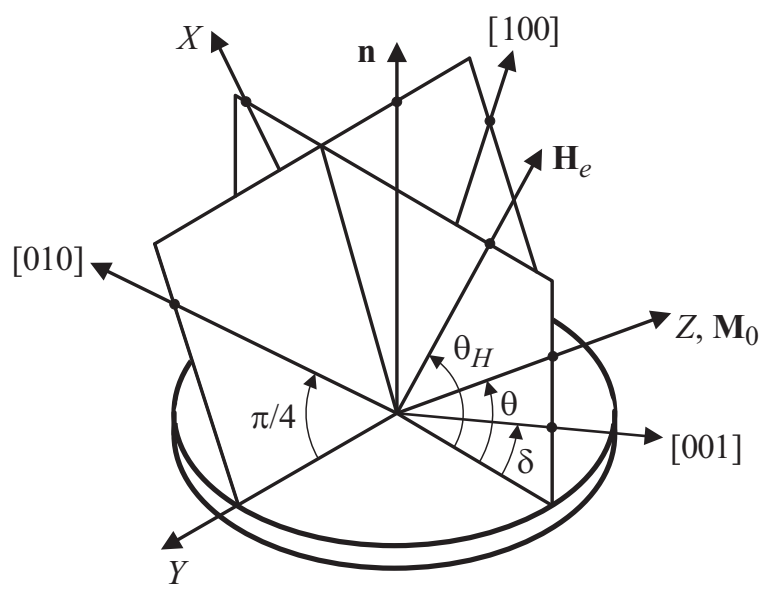

Рис. 1. Модель пленки. 
него намагничивающего поля, $\mathbf{n}-$ нормаль к пленке, $X, Y, Z-$ координатные оси, причем $Z \| \mathbf{M}_{0}$ и $Y \perp \mathbf{n}$, $\theta, \theta_{H}, \delta-$ углы наклона к пленке векторов $\mathbf{M}_{0}$, $\mathbf{H}_{e}$ и кристаллографической оси [001] соответственно. Предполагалось, что векторы $\mathbf{H}_{e}$ и $\mathbf{M}_{0}$ расположены в плоскости кристаллографической симметрии, проходящей через ось [001]. Данное предположение позволяет сократить количество угловых переменных, используемых в модели.

Плотность энергии магнитной анизотропии в большинстве пленок ферритов с кубической симметрией кристаллической решетки, выращенных на монокристаллических подложках, описывается выражением [1]

$$
W_{a}=-\frac{K_{c 1}}{2 M_{0}^{4}} \sum_{\mathrm{p}} M_{p}^{4}+K_{u 1} \cos ^{2} \theta,
$$

где $K_{c 1}$ - первая константа кубической магнитной анизотропии, и суммирование производится по проекциям вектора намагниченности на оси системы координат, образованной осями симметрии кристалла четвертого порядка, $K_{u 1}-$ первая константа одноосной нормальной анизотропии. Данное выражение было использовано при выводе формулы для частоты ФМР, а также при выводе уравнения связи между углами $\theta$ и $\theta_{H}$. При этом поля анизотропии определены выражениями $H_{c}=K_{c 1} / M_{0}$ и $H_{u}=2 K_{u} / M_{0}$.

Частота ФМР в рассматриваемой модели задана формулой [5]:

$$
\begin{aligned}
\left(f_{0} / g\right)^{2}= & \left(H_{e z}+H_{c} n_{x}+4 \pi M_{\mathrm{eff}} \cos 2 \theta\right) \\
& \times\left(H_{e z}+H_{c} n_{y}-4 \pi M_{\mathrm{eff}} \sin ^{2} \theta\right),
\end{aligned}
$$

где $g$ - гиромагнитное отношение (в расчетах полагалось $g=2.8 \mathrm{MHz} / \mathrm{Oe}), H_{e z}$ - проекция вектора $\mathbf{H}_{e}$ на вектор $\mathbf{M}_{0}, 4 \pi M_{\text {eff }}=4 \pi M_{0}-H_{u}-$ эффективная намагниченность, параметры $n_{x}$ и $n_{y}$ учитывают влияние кристаллографической магнитной анизотропии и определены формулами

$$
\begin{gathered}
n_{x}=\frac{1}{2}[\cos 2(\theta-\delta)+3 \cos 4(\theta-\delta)], \\
n_{y}=\frac{1}{8}[-3+16 \cos 2(\theta-\delta)+3 \cos 4(\theta-\delta)] .
\end{gathered}
$$

В общем случае частота ФМР определяется значениями магнитных параметров феррита $4 \pi M_{\mathrm{eff}}, H_{c}$ и значениями параметров вектора напряженности намагничивающего поля $H_{e}, \theta_{H}$. Разрабатываемая модель направлена на решение задачи устойчивости частоты к изменениям магнитных параметров феррита. Из формулы (2) можно вывести соотношение, связывающее между собой малые изменения квадрата частоты с изменениями этих параметров. В линейном приближении соотношение будет иметь следующий вид:

$$
\delta\left(\frac{f_{0}}{g}\right)^{2}=4 \pi M_{\mathrm{eff}}\left[A \delta\left(4 \pi M_{\mathrm{eff}}\right)+B \delta H_{c}\right],
$$

где $A$ и $B$ - безразмерные величины, задаваемые полными производными:

$$
\begin{gathered}
A=\frac{1}{4 \pi M_{\mathrm{eff}}} \frac{d\left(f_{0} / g\right)^{2}}{d\left(4 \pi M_{\mathrm{eff}}\right)}, \\
B=\frac{1}{4 \pi M_{\mathrm{eff}}} \frac{d\left(f_{0} / g\right)^{2}}{d H_{c}} .
\end{gathered}
$$

При выводе выражений для производных должна быть учтена зависимость $\theta, H_{e z}, n_{x}, n_{y}$ от $4 \pi M_{\mathrm{eff}}, H_{c}, H_{e}$, $\theta_{H}$. Необходимые соотношения могут быть выведены из уравнений, определяющих проекции намагничивающего поля $\mathbf{H}_{e}$ на координатные оси $Z$ и $X$ :

$$
\begin{gathered}
H_{e z}=H_{e} \cos \left(\theta_{H}-\theta\right), \\
H_{e x}=H_{e} \sin \left(\theta_{H}-\theta\right)=M_{0} N_{x z}^{c}+\left(\frac{\sin 2 \theta}{2}\right) 4 \pi M_{\text {eff. }}
\end{gathered}
$$

Здесь $N_{x z}^{c}$ компонента эффективного размагничивающего фактора кубической анизотропии, определяемая формулой [5]:

$$
M_{0} N_{x z}^{c}=\frac{1}{8} H_{c}[2 \sin 2(\theta-\delta)+3 \sin 4(\theta-\delta)] .
$$

Из уравнений (6)-(8) следуют уравнения связи для вариаций $\delta \theta=C \delta\left(4 \pi M_{\mathrm{eff}}\right)+D \delta H_{c}$ и $\delta H_{e z}=K \delta \theta$, где

$$
\begin{gathered}
C=-\frac{\sin 2 \theta}{2 H_{e z}+H_{c}[\cos 2(\theta-\delta)+3 \cos 4(\theta-\delta)]+} \\
+2 \cdot 4 \pi M_{\mathrm{eff}} \cos 2 \theta \\
D=-\frac{2 \sin 2(\theta-\delta)+3 \sin 4(\theta-\delta)}{4\left\{2 H_{e z}+H_{c}[\cos 2(\theta-\delta)+3 \cos 4(\theta-\delta)]+\right.} \\
\left.+2 \cdot 4 \pi M_{\mathrm{eff}} \cos 2 \theta\right\}
\end{gathered}
$$

$K=\frac{H_{c}}{8}[2 \sin 2(\theta-\delta)+3 \sin 4(\theta-\delta)]+\frac{4 \pi M_{\mathrm{eff}}}{2} \sin 2 \theta$.

Из равенств (3) и (4) следует $\delta n_{x}=\left(\partial n_{x} / \partial \theta\right) \delta \theta$ и $\delta n_{y}=\left(\partial n_{y} / \partial \theta\right) \delta \theta$.

Таким образом, расчетные формулы для $A$ и $B$ будут иметь вид

$$
\begin{aligned}
4 \pi M_{\mathrm{eff}} A= & \frac{\partial\left(f_{0} / g\right)^{2}}{\partial\left(4 \pi M_{\mathrm{eff}}\right)}+\left[\frac{\partial\left(f_{0} / g\right)^{2}}{\partial H_{e z}} K+\frac{\partial\left(f_{0} / g\right)^{2}}{\partial \theta}\right. \\
& \left.+\frac{\partial\left(f_{0} / g\right)^{2}}{\partial n_{x}} \frac{\partial n_{x}}{\partial \theta}+\frac{\partial\left(f_{0} / g\right)^{2}}{\partial n_{y}} \frac{\partial n_{y}}{\partial \theta}\right] C, \\
4 \pi M_{\mathrm{eff}} B= & \frac{\partial\left(f_{0} / g\right)^{2}}{\partial H_{c}}+\left[\frac{\partial\left(f_{0} / g\right)^{2}}{\partial H_{e z}} K+\frac{\partial\left(f_{0} / g\right)^{2}}{\partial \theta}\right. \\
& \left.+\frac{\partial\left(f_{0} / g\right)^{2}}{\partial n_{x}} \frac{\partial n_{x}}{\partial \theta}+\frac{\partial\left(f_{0} / g\right)^{2}}{\partial n_{y}} \frac{\partial n_{y}}{\partial \theta}\right] D,
\end{aligned}
$$




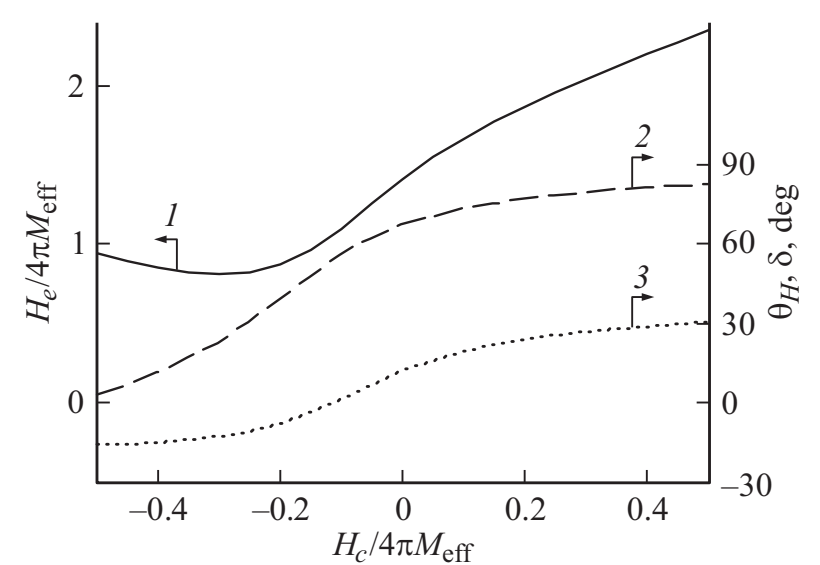

Рис. 2. Зависимости оптимальных значений $H_{e} / 4 \pi M_{\text {eff }}(1)$, $\theta_{H}(2)$ и $\delta(3)$ от $H_{c} / 4 \pi M_{\text {eff. }}$.

Конечная цель расчетов заключается в том, чтобы из уравнений $A=0, B=0$ и (2) определить значения параметров $\delta, \theta$ и $H_{e z}$. При этом в уравнение (2) нужно подставить значение стабилизируемой частоты $f_{0}$, а в правые части равенств (12), (13) подставить исходные значения $4 \pi M_{\mathrm{eff}}$ и $H_{c}$. После определения значений $\delta, \theta$ и $H_{e z}$ нужно выполнить переход к значениям экспериментально контролируемых параметров $\theta_{H}$ и $H_{e}$. Такой переход основывается на уравнениях (6)-(8).

В качестве примера на рис. 2 приведены результаты расчетов, выполненных для случая $f_{0}=4 \pi M_{\text {eff }} g$. Исходные значения $H_{c}$ и $4 \pi M_{\text {eff }}$ отложены по оси абсцисс в виде отношения $H_{c} / 4 \pi M_{\text {eff. }}$ Уместно отметить, что в настоящее время практическое применение нашли пленки ферритов со слабой анизотропией, когда $\left|H_{c}\right| \ll 4 \pi M_{\text {eff. }}$ Использованный в расчетах интервал значений $H_{c} / 4 \pi M_{\mathrm{eff}}$ позволяет сделать вывод о том, что оптимальные значения параметров $\delta, \theta_{H}$ и $H_{e}$ могут быть найдены и для перспективных материалов, у которых $H_{c}$ будет сравнимым с $4 \pi M_{\text {eff. }}$

\section{Анализ результатов моделирования}

В рассматриваемой задаче критерием оптимальности выбора значений параметров $H_{e}, \theta_{H}, \delta$ является обращение в нуль выражения для $\delta f_{0}$, полученного в линейном по вариациям $\delta\left(4 \pi M_{\text {eff }}\right)$ и $\delta H_{c}$ приближении. Тогда $\delta f_{0}$ будет задано квадратичной формой

$$
\begin{aligned}
& \frac{\delta f_{0}}{f_{0}}=\frac{1}{4\left(f_{0} / g\right)^{2}}\left\{\frac{d^{2}\left(f_{0} / g\right)^{2}}{d\left(4 \pi M_{\mathrm{eff}}\right)^{2}}\left[\delta\left(4 \pi M_{\mathrm{eff}}\right)\right]^{2}\right. \\
& \left.+2 \frac{d^{2}\left(f_{0} / g\right)^{2}}{d H_{c} d\left(4 \pi M_{\mathrm{eff}}\right)} \delta\left(4 \pi M_{\mathrm{eff}}\right) \delta H_{c}+\frac{d^{2}\left(f_{0} / g\right)^{2}}{d H_{c}^{2}}\left(\delta H_{c}\right)^{2}\right\} .
\end{aligned}
$$

Расчет коэффициентов квадратичной формы удобно выполнить исходя из формулы (2) с подстановками в нее вместо параметров $H_{e z}, n_{x}, n_{y}$ выражений (3), (4), (6). При этом в выражениях надо использовать найденные значения $H_{e}, \theta_{H}$ и $\delta$. Правая часть формулы (2) после такой подстановки будет определена параметрами $4 \pi M_{\mathrm{eff}}$, $H_{c}, \theta$, причем, как уже было отмечено, независимыми будут только параметры $4 \pi M_{\text {eff }}$ и $H_{c}$. Тогда вторые производные в коэффициентах квадратичной формы нужно рассчитывать по формулам

$$
\begin{gathered}
\frac{d^{2}\left(f_{0} / g\right)^{2}}{d\left(4 \pi M_{\mathrm{eff}}\right)^{2}}=\frac{\partial\left[\frac{d\left(f_{0} / g\right)^{2}}{d\left(4 \pi M_{\mathrm{eff}}\right)}\right]}{\partial\left(4 \pi M_{\mathrm{eff}}\right)}+\frac{\partial\left[\frac{d\left(f_{0} / g\right)^{2}}{d\left(4 \pi M_{\mathrm{eff}}\right)}\right]}{\partial \theta} \frac{d \theta}{d\left(4 \pi M_{\mathrm{eff}}\right)}, \\
\frac{d^{2}\left(f_{0} / g\right)^{2}}{d H_{c} d\left(4 \pi M_{\mathrm{eff}}\right)}=\frac{\partial\left[\frac{d\left(f_{0} / g\right)^{2}}{d\left(4 \pi M_{\mathrm{eff}}\right)}\right]}{\partial H_{c}}+\frac{\partial\left[\frac{d\left(f_{0} / g\right)^{2}}{d\left(4 \pi M_{\mathrm{eff}}\right)}\right]}{\partial \theta} \frac{d \theta}{d H_{c}}, \\
\frac{d^{2}\left(f_{0} / g\right)^{2}}{d H_{c}^{2}}=\frac{\partial\left[\frac{d\left(f_{0} / g\right)^{2}}{d H_{c}}\right]}{\partial H_{c}}+\frac{\partial\left[\frac{d\left(f_{0} / g\right)^{2}}{d H_{c}}\right]}{\partial \theta} \frac{d \theta}{d H_{c}} .
\end{gathered}
$$

В этих формулах первые производные также должны быть рассчитаны с учетом зависимости $\theta$ от $4 \pi M_{\text {eff }}$ и $H_{c}$ :

$$
\begin{gathered}
\frac{d\left(f_{0} / g\right)^{2}}{d\left(4 \pi M_{\mathrm{eff}}\right)}=\frac{\partial\left(f_{0} / g\right)^{2}}{\partial\left(4 \pi M_{\mathrm{eff}}\right)}+\frac{\partial\left(f_{0} / g\right)^{2}}{\partial \theta} \frac{d \theta}{d\left(4 \pi M_{\mathrm{eff}}\right)}, \\
\frac{d\left(f_{0} / g\right)^{2}}{d H_{c}}=\frac{\partial\left(f_{0} / g\right)^{2}}{\partial H_{c}}+\frac{\partial\left(f_{0} / g\right)^{2}}{\partial \theta} \frac{d \theta}{d H_{c}} .
\end{gathered}
$$

Производные $d \theta / d\left(4 \pi M_{\mathrm{eff}}\right)$ и $d \theta / d H_{c}$ определены правыми частями соответственно выражений (9) и (10) с подстановками в них вместо $H_{e z}$ выражения (6).

На рис. 3 приведены примеры зависимостей, задаваемых квадратичной формой. Рассчитанные значения $\delta f_{0}$ расположены на поверхностях гиперболических параболоидов, имеющих форму седла. Из сравнения поверхностей между собой видно, что зависимость частоты от вариаций магнитных параметров уменьшается

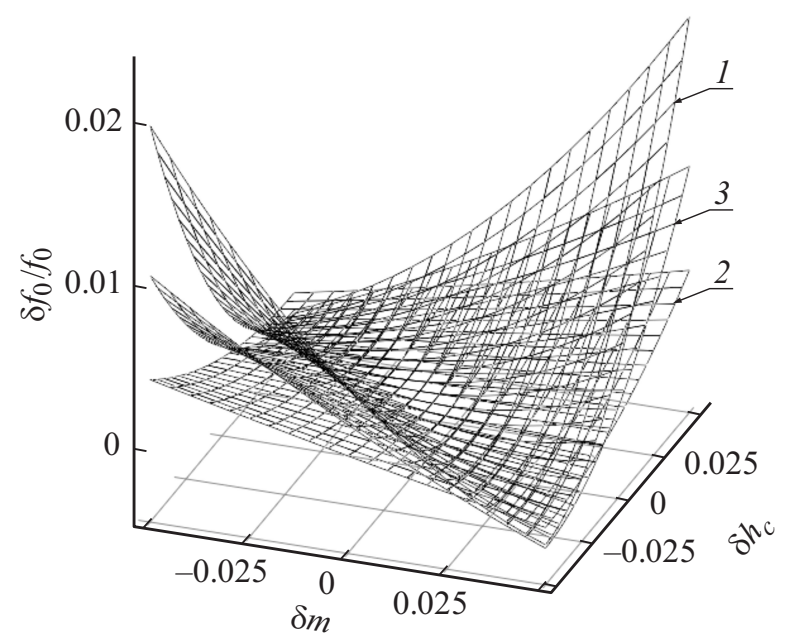

Рис. 3. Относительное смещение частоты ФМР в пленках с оптимизированными параметрами. Исходные значения поля анизотропии: $1-H_{c} /\left(4 \pi M_{\mathrm{eff}}\right)=0.5,2-H_{c} /\left(4 \pi M_{\mathrm{eff}}\right)=$ $=-0.5,3-H_{c}=0$. Исходное значение частоты ФМР: $f_{0}=4 \pi M_{\text {eff }} g$. Обозначения осей: $\delta m \equiv \delta\left(4 \pi M_{\text {eff }}\right) /\left(4 \pi M_{\text {eff }}\right)$ и $\delta h_{c} \equiv \delta H_{c} /\left(4 \pi M_{\text {eff }}\right)$. 


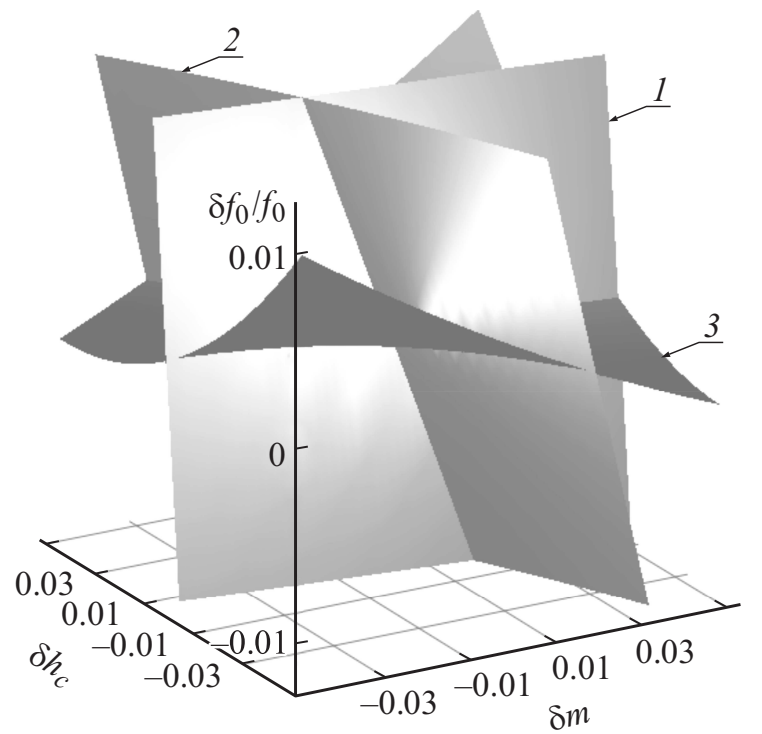

Рис. 4. Относительное смещение частоты ФИР: 1 пленка $\{110\}$ и $\theta_{H}=0,2-$ пленка $\{110\}$ и $\theta_{H}=\pi / 2$, 3 - пленка с оптимизированными параметрами. Исходные значения: $f_{0}=4 \pi M_{\text {eff }} g, H_{c}=0$. Обозначения осей: $\delta m \equiv \delta\left(4 \pi M_{\text {eff }}\right) /\left(4 \pi M_{\text {eff }}\right)$ и $\delta h_{c} \equiv \delta H_{c} /\left(4 \pi M_{\text {eff }}\right)$.

с уменьшением $H_{c}$ и с переходом $H_{c}$ от положительных значений к отрицательным.

Также представляет интерес сравнение оптимизированных пленок с „обычными“. Для такого сравнения был выполнен расчет, результаты которого представлены на рис. 4. Наряду с оптимизированной была рассмотрена пленка с кристаллографической ориентацией типа $\{110\}$, намагниченная двумя способами - касательно и перпендикулярно. В пленках обоего типа поле $H_{c}$ варьировало вблизи значения $H_{c}=0$, и, как и в предыдущем расчете (рис. 3), полагалось $f_{0}=4 \pi M_{\text {eff }}$. По-прежнему была использована модель, изображенная на рис. 1. Пленка, взятая для сравнения, была ориентирована вдоль плоскости (011) и характеризовалась значением $\delta=0$. Кроме того, полагалось $\theta_{H}=0$ при касательном намагничивании и $\theta_{H}=\pi / 2-$ при перпендикулярном. Кристаллографическая симметрия рассматриваемых направлений намагничивания позволяет полагать $\theta=\theta_{H}$ и не учитывать вариаций $\delta H_{e z}, \delta n_{x}, \delta n_{y}, \delta \theta$. Данное утверждение также следует и из выражений (6)-(11). Расчет на основе формул (2)-(4) и (6) приводит к следующим соотношениям:

$$
\begin{aligned}
& \left(\frac{\delta f_{0}}{f_{0}}\right)_{\tan }= \\
& \left.=\sqrt{\times\left[\sqrt{1.25}-0.5+\frac{2 \delta H_{c}}{4 \pi M_{\mathrm{eff}}}\right) \times}+0.5+\frac{\delta\left(4 \pi M_{\mathrm{eff}}\right)}{4 \pi M_{\mathrm{eff}}}+\frac{2 \delta H_{c}}{4 \pi M_{\mathrm{eff}}}\right]
\end{aligned}
$$

в случае касательного намагничивания и

$$
\begin{aligned}
& \left(\frac{\delta f_{0}}{f_{0}}\right)_{\mathrm{per}}= \\
& \sqrt{\left[1-\frac{\delta\left(4 \pi M_{\mathrm{eff}}\right)}{4 \pi M_{\mathrm{eff}}}+\frac{\delta H_{c}}{\left.4 \pi M_{\mathrm{eff}}\right]\left[1-\frac{\delta\left(4 \pi M_{\mathrm{eff}}\right)}{4 \pi M_{\mathrm{eff}}}-\frac{2 \delta H_{c}}{4 \pi M_{\mathrm{eff}}}\right]}\right.}-1
\end{aligned}
$$

в случае перпендикулярного намагничивания.

Как следует из рисунка, рассчитанные по данным уравнениям поверхности имеют форму, близкую к плоскостям, задаваемым линейными зависимостями между вариациями $\delta f_{0}, \delta\left(4 \pi M_{\mathrm{eff}}\right)$ и $\delta H_{c}$. Эти зависимости можно получить, представляя правые части формул (14), (15) линейными по вариациям $\delta\left(4 \pi M_{\mathrm{eff}}\right), \delta H_{c}$ приближениями. В оптимизированной пленке зависимость $\delta f_{0}$ от $\delta\left(4 \pi M_{\text {eff }}\right)$ и $\delta H_{c}$ является квадратичной, и при этом поверхность, описываемая квадратичной формой, демонстрирует более высокую стабильность частоты, чем плоскости, задаваемые линейными зависимостями.

\section{Заключение}

Предложенный способ стабилизации может быть применен и к частотам из спектра магнитостатических волн. Отличия от ФМР будут лишь в выражении для частоты - в случае волны вместо формулы (2) надо исходить из дисперсионного уравнения с подстановкой в него значения волнового числа.

Результаты работы могут быть использованы при разработке устройств спин-волновой электроники. В настоящее время основными материалами данного класса устройств являются пленки железоиттриевого граната (ЖИГ, $\mathrm{Y}_{3} \mathrm{Fe}_{5} \mathrm{O}_{12}$ ), выращенные на монокристаллических подложках гадолиний-галлиевого граната $\left(\mathrm{Gd}_{3} \mathrm{Ga}_{5} \mathrm{O}_{12}\right)$. Пленкам ЖИГ присуща кубическая магнитная анизотропия. Намагниченностью насыщения и полем кубической анизотропии ЖИГ можно управлять, замещая ионы железа ионами галлия и скандия [6-8]. Нестабильность спектров магнитных колебаний таких пленок связана с зависимостью магнитных параметров от температуры и от химического состава. Метод, описанный в настоящей работе, позволяет минимизировать влияние обоих факторов и тем самым стабилизировать частотные характеристики устройств. Кроме того, в методе не вводились ограничения на значения магнитных параметров феррита, так что построенная теория может быть применена и к новым разрабатываемым материалам.

\section{Список литературы}

[1] Гуревич А.Г., Мелков Г.А. Магнитные колебания и волны. М.: Наука, 1994. 464 с.

[2] Шагаев В.В. // ЖТФ. 1998. Т. 6. Вып. 10. С. 99-103.

[3] Шагаев В.В. // ФТТ. 2003. Т. 45. Вып. 12. С. 2215-2221.

[4] Шагаев В.В. // Письма в ЖТФ. 2006. Т. 32. Вып. 18. С. 1-6. 
[5] Бондаренко Г.Г., Шагаев В.В. // Изв. вузов. Сер. Физика. 2013. T. 56. № 1/2. C. 13-22.

[6] Казаков Г.Т., Маряхин А.В., Нам Б.П., Сухарев А.Г., Филимонов Ю.А., Шеин И.В., Суров Ю.И., Марголина Р.Ю. // Письма в ЖТФ. 1988. Т. 14. Вып. 19. С. 1733-1736.

[7] Hansen P., Röschmann P., Tolksdorf W. // J. Appl. Phys. 1974. Vol. 45. N 6. P. 2728-2732.

[8] Hansen P. // J. Appl. Phys. 1974. Vol. 45. N 8. P. 3638-3642. 